\title{
Espacio muerto en lactantes normales y con patología pulmonar
}

\author{
Dras. Yerka Gercia, ${ }^{* *}$ Silvia Back, ${ }^{*}$ Carmen Lisboa." ${ }^{* *}$
}

\begin{abstract}
In this paper we describe the equipment and technics used to study in infants the ratio between physiological dead space and tidal volume, as a measurement of this efficiency of pulmonary ventilation.

The usefulness of this procedure is proven in ten normal infants and ten infants with different pathologr.

In normal infants. the results demonstrate co be very similar to the values found in the aduite $130 \%$ ). In infants with pulmonary disfunction, the ratio was found altered according to the respiratory pathology.
\end{abstract}

Las técnicas necesarias para el estudio de la función respiratoria en lactantes son restringidas. Las limitaciones que representan la adaptación del instrumental al tamano y características del sistema respiratorio del niño, unido a la falta de cooperación del paciente hacen que estas pruebas sean relativamente complicadas. Por lo tanto toda metodología que permita el estudio de la función pulmonar en lactantes, representa siempre un avance en esta materia.11. 21

F.l objetivo de esle trabajo ha sido adaptar en lactantes la técnica e instrumental necesarios para medir la efectividad de la ventilación pulmonar mediante la medición de la relación entre espacio muerto respiratorio y volumen corriente. ${ }^{3}, 4.5$.

\section{MATERIAI, Y METODO}

El estudio se realizó en 11 lactantes normales cuyas edades fluctuaron entre 4 y 17 meses con un promedio de $13.19=6.59$ m y en otros 10 . con diferentes enfermedades respiratorias cuyas caracteristicas clínicas se encuentran detalladas en la tabla N." 1 .

La determinación del espacio muerto físiolígico se realizí de acuerdo a la fórmula propuesta por Bohr, que establece que el volumen de gas expirado consiste en una mezcla de gas proveniente del espa-

* Servicio Broncopulrovnar, Hospital Luí ('alvo Mackenna.

** Quimié-Farmacéutico, Laburaturio Centro CardiovascuLar, Horspital Lujs Calvi, Mackenna.

*F* Servicio Cordiurrespirstoriv. Universidad tiatrilica.
Tabla N." 1

CARACTERISTICAS CLINICAS DEL GRUPO DE LACTANTES CON PATOLOGIA RESPIRATORIA

\begin{tabular}{clc} 
N." Caso & DIAGNOSTICO & EDAD \\
\hline 1 & Atelectasia bilateral & $8 \mathrm{~m}$ \\
2 & Pulmón poliquístico & $7 \mathrm{~m}$ \\
& Derrame pleural & $15 \mathrm{~m}$ \\
4 & Atelectasia pulmón derecho & $6 \mathrm{~m}$ \\
5 & Neumonitis & $11 \mathrm{~m}$ \\
6 & Absceso insuflado & $4 \mathrm{~m}$ \\
7 & Neumatocele & $12 \mathrm{~m}$ \\
8 & Enfisema lubar & $5.5 \mathrm{~m}$ \\
9 & Enfisema lobar & $2.5 \mathrm{~m}$ \\
10 & Enfisema bilateral & $11 \mathrm{~m}$
\end{tabular}

$T=10$ casos $\overline{\mathrm{x}}=7.54=3.73$

cio muerto y de los alvéolos, empleando $\mathrm{CO}_{2}$ como gas de prueba. $(4,5,6 \mathrm{r}$

$$
\begin{aligned}
& V_{\mathrm{D}}=\mathrm{FACO}_{2}-\mathrm{FExCO}_{2} \\
& v_{\mathrm{T}} \\
& \frac{\mathrm{FACO}_{2}}{V_{\mathrm{T}}}=\frac{\mathrm{PACO}_{2}-\mathrm{PExCO}_{2}}{\mathrm{PACO}_{2}}
\end{aligned}
$$


La $\mathrm{FexCO}_{2}$ se determino reongiendo las muesras de gas expirado en una holsa de látex por medio de una valvula nasal descrita en un trabajo anleriortos, Lasada en el medelo de Cralinke y hudolph. ' ' I a concentrarión de (.O2 de las muestras se determini en un analizador de gases de Scholander.: $x$,

Todas estas pruebas se realizarim con los niños dormidos para lor cual fue necesario la administracicin de secobarbital en dosis de $7.5 \mathrm{mg}$ por $\mathrm{Kg}$ de pesin.

Para conocer el valor de la FACO2. se considerio la $\mathrm{PCO} 2$ arterial similar a la $\mathrm{PC} \mathrm{CO}_{2}$ alveular, basándıs: en el principio que existe un equilibrio de $\mathrm{PCO}_{2}$ entre aire alveolar $y$ capilar pulmonar en pacientes que no paseen un corlac ircuituarterio-ve'noser significativa."51 Las muestras arterializadas se tomaron en forma simultánea a la reopleceión de gas expirado. las que se ubluvieron en capilates Radiometer por punción del talín. previos calentamienter de pierna y pie a $45^{\text {"1 }}$ C durante 10 min. ${ }^{\text {9. El }}$ El valor de PCO2 arterial se determinio en un equipa Radiumetér empleandu el métodu de] cequilibrin y calculándolo por medio del Nomograma de Siggaard-Andersen. 110.11 ,

\section{RESI:LTADOS}

Lus valores cheontrados an los lactantes normales y cron patolengía pulmonar están detalladus en los gráficus N.os l y 2, a partir de los cuales se puede punIualizar:

RELACION YDS/NT EN ZAOTHATES

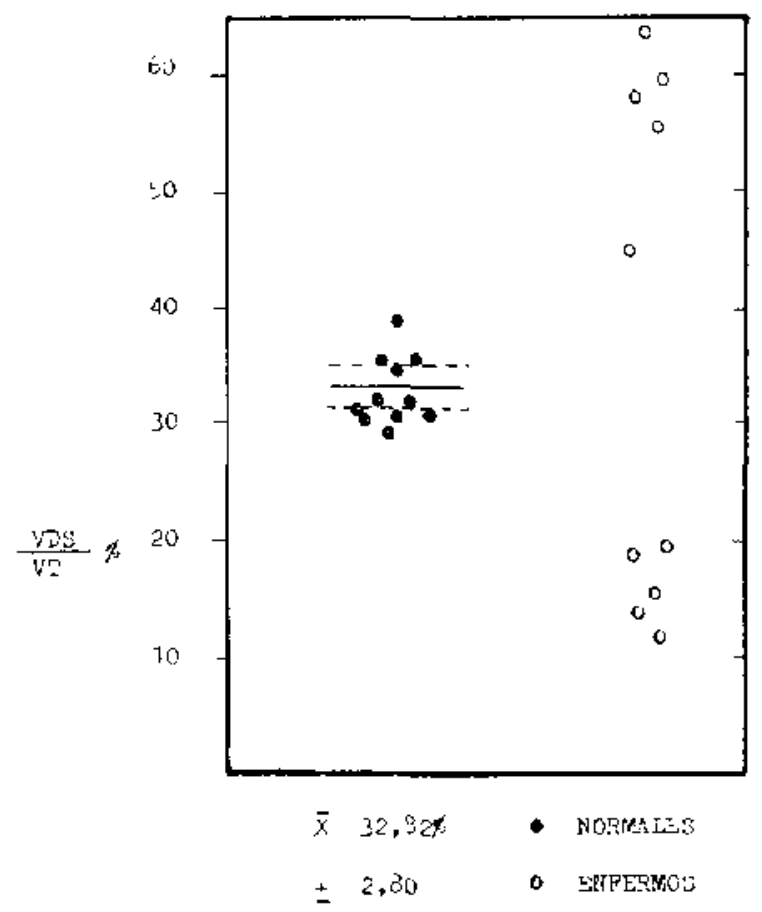

Gzófleo HQ1

VENTILACION PULMONAR EN LACTANTES
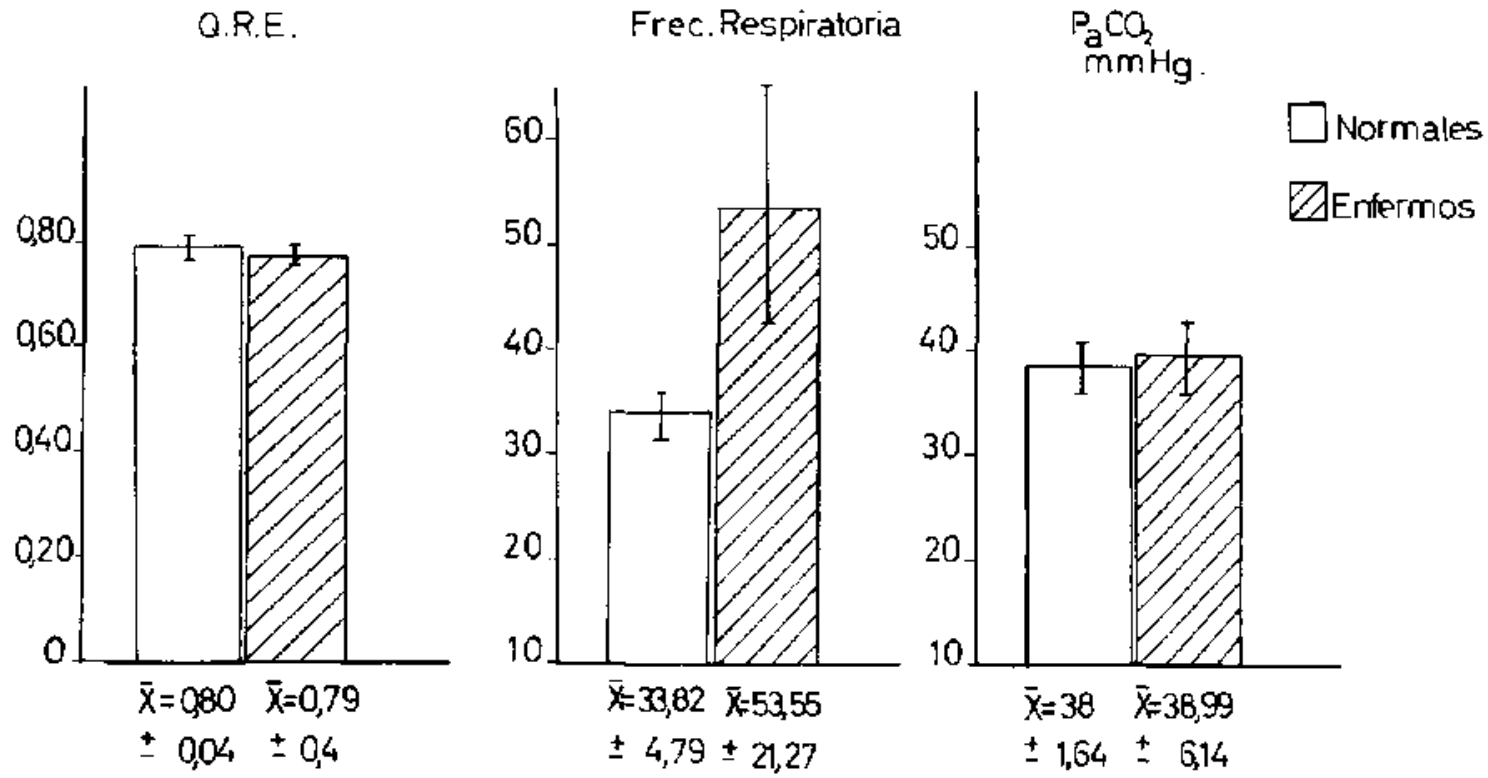